\title{
Persistent trismus following mandibular third molar extraction and its management: A case report and literature review
}

\author{
YINGJIE ZHANG $^{1 *}$, PEILIN ZHUANG ${ }^{2 *}$, BO JIA ${ }^{3 *}$, JING XU $^{3}$, QIUJU CUI ${ }^{3}$, \\ LIHUAN NIE ${ }^{4}$, ZHIPING WANG ${ }^{3}$ and ZHAOQIANG ZHANG ${ }^{3}$ \\ ${ }^{1}$ Department of Orthodontics, Stomatological Hospital, Southern Medical University, Guangzhou, \\ Guangdong 510280; ${ }^{2}$ Department of Stomatology, Sun Yat-Sen Memorial Hospital, Sun Yat-Sen University, \\ Guangzhou, Guangdong 510120; ${ }^{3}$ Department of Oral and Maxillofacial Surgery, Stomatological Hospital, \\ Southern Medical University, Guangzhou, Guangdong 510280; ${ }^{4}$ Department of Pediatric Dentistry, Haizhu Square Branch, \\ Stomatological Hospital, Southern Medical University, Guangzhou, Guangdong 510120, P.R. China
}

Received June 18, 2020; Accepted November 5, 2020

DOI: $10.3892 /$ wasj.2020.73

\begin{abstract}
Trismus is one of the common complications which occur following the extraction of mandibular impacted third molars. This generally occurs due to low-grade infection, repeated muscle stimulation, as well as other causes. This symptom is usually relieved after 1 to 2 weeks; however, it may persist for $>1$ month post-operatively in very rare cases. The present study reports the case of a patient with trismus at 45 days following mandibular third molar extraction. The patient received local and systemic anti-inflammatory treatment, combined with incision and drainage therapy under local anesthesia. In the present study, an analysis of the factors associated with the occurrence and development of trismus is also included, as well as appropriate management strategies in order to provide an effective treatment method for affected patients and for the prevention of trismus in the future.
\end{abstract}

\section{Introduction}

The extraction of mandibular impacted third molars, a routine outpatient surgery performed by oral and maxillofacial surgeons, is associated with common complications, such as trismus, pain and infection (1). The most severe cases of trismus usually occur at 2 days post-operatively. The primary causes include elevation of flap beyond the external oblique

Correspondence to: Dr Zhaoqiang Zhang or Dr Zhiping Wang, Department of Oral and Maxillofacial Surgery, Stomatological Hospital, Southern Medical University, 366 South of Jiangnan Boulevard, Guangzhou, Guangdong 510280, P.R. China

E-mail: 187234415@smu.edu.cn

E-mail:sum20004218@163.com

*Contributed equally

Key words: trismus, impacted third molars, prevention, treatment ridge, low-grade infection following local anesthesia and repeated stimulation of the medial pterygoid muscle (inferior alveolar nerve block), as well as other causes (2). The symptom of trismus is alleviated by the post-operative local injection of dexamethasone $(3,4)$. Generally, trismus is gradually alleviated or disappears within approximately 1 to 2 weeks post-operatively; however, in very rare cases, trismus persists for $>1$ month. The present study reports the case of a patient who exhibited trismus for 45 days following mandibular third molar extraction. The patient received local and systemic anti-inflammatory treatment, as well as incision and drainage therapy under local anesthesia. Furthermore, the factors associated with the occurrence and development of trismus were also analyzed, and appropriate management strategies are discussed in order to provide an effective treatment method for affected patients, as well as to prevent the occurrence of trismus in the future.

\section{Case report}

Initial presentation and treatment. A 30-year-old male patient visited the Department of Oral and Maxillofacial Surgery, the Stomatological Hospital, Southern Medical University (Guangzhou, China) for the treatment of edema and pain around the crown of the left third molar that had lasted for $>6$ months. Informed consent was obtained from the patient for his participation in the study and for publishing the relevant clinical data. A clinical examination revealed that tooth $38 \mathrm{had}$ partly erupted and was covered by a distal gingiva flap, which was slightly red and swollen. The surface caries of tooth 38 extended to the dentin layer and were accompanied by transient sensitivity to cold stimulation. There were no abnormalities in terms of probing, percussion, mobility, or mouth opening; panoramic X-ray imaging revealed the mesioangular impaction of tooth 38 (Fig. 1, white rectangular region) and distocervical low density in the area of tooth 37 (Fig. 1, red arrow). On the day of the initial visit, according to the guidelines for the treatment of mandibular third molars, patient symptoms are designated as present and attributable to the third molar (5), the extraction of the affected tooth was performed following iodophor 


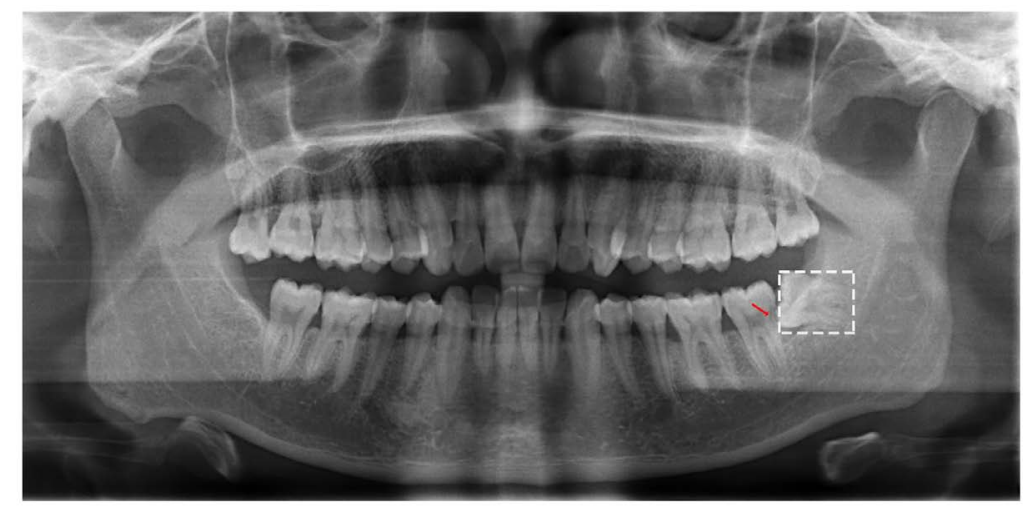

Figure 1. Panoramic X-ray imaging showing mesioangular impaction of tooth 38 (white rectangular region) and distocervical low density in the area of tooth 37 (red arrow).
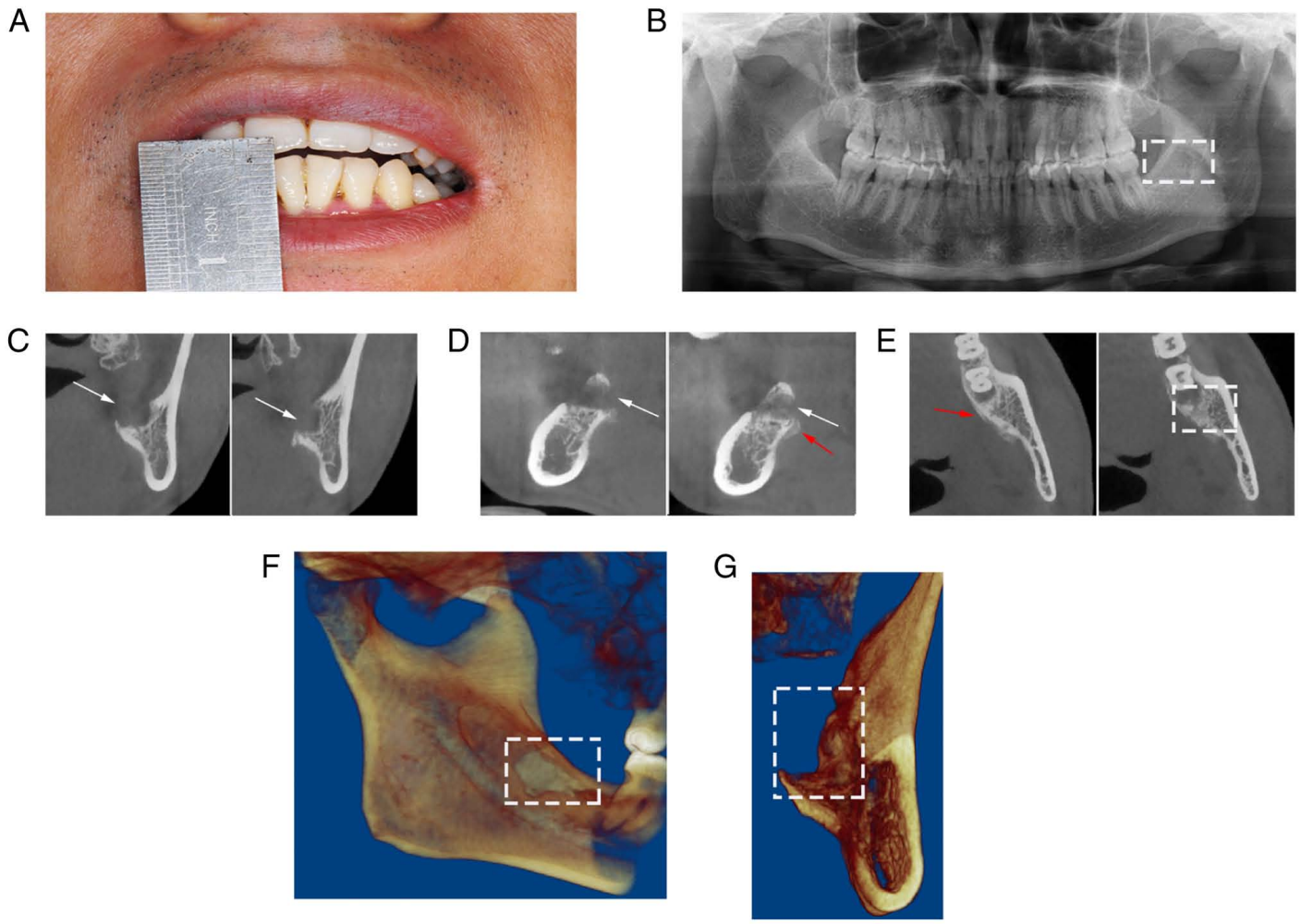

Figure 2. (A) The extent of mouth opening was approximately $2 \mathrm{~mm}$ at 30 days postoperatively. (B) Panoramic X-ray imaging demonstrating reduced bone density in the left mandible (white rectangular region) and tight occlusion of anterior teeth without a gap. (C-E) Cone beam computed tomography could not clearly discern the repair to bone in the alveolar fossa of tooth 38 . The lingual bone plate in the root tip area was missing (C and D, white arrows) and 3-dimensional reconstruction of $\mathrm{CBCT}$ images also revealed the absence of the lingual bone plate ( $\mathrm{F}$ and $\mathrm{G}$, white rectangular region); moreover, obvious lamellar periosteal hyperplasia was present in the corresponding area ( $\mathrm{D}$ and $\mathrm{E}$, red arrows). Some areas of the lingual bone plate were slightly rough (E, white rectangular region), and the soft tissue gap was difficult to discern in the bottom of the left side of the mouth. These radiologic features indicated marginal osteomyelitis of the left mandible.

disinfection in the area of tooth 38 . Under inferior alveolar nerve block and infiltration anesthesia (methylpiperazine hydrochloride/epinephrine), the flap was turned and a dynamic bone drilling system was used to separate the crown from the root. Thus, tooth 38 was extracted and the alveolar fossa was scraped to remove inflamed tissue. Normal saline was used to rinse the socket and the wound was closed with tight stitching to achieve hemostasis. During the surgery, both the tooth and lingual bone plate was removed together as the tooth root was adhered to the lingual bone plate. The extraction procedure lasted for approximately $30 \mathrm{~min}$; the patient was instructed to take anti-inflammatory $(0.375 \mathrm{~g}$ cefaclor sustained-release tablets) and detumescence ( $0.75 \mathrm{mg}$ dexamethasone tablets) drugs for 3 days post-operatively.

Occurrence of trismus. At 8 days post-operatively, the patient returned for a routine follow-up. A clinical examination revealed slight trismus (the distance of the incisal edges between the upper and lower incisor was approximately $28 \mathrm{~mm}$ ). No obvious edema or pain were observed in the surgical area and normal wound healing was observed. The sutures were removed following iodophor disinfection and the wound 
Table I. Routine blood examination results.

\begin{tabular}{lccc}
\hline Parameter & Value & Reference range & Unit \\
\hline White blood cell count & 7.6 & $3.5-9.5$ & $10^{9} / 1$ \\
Lymphocyte count & 2.4 & $0.8-4.0$ & $10^{9} / 1$ \\
Neutrophil count & 0.3 & $0.1-1.5$ & $10^{9} / 1$ \\
Neutrophilic granulocyte count & 4.9 & $2.0-7.0$ & $10^{9} / 1$ \\
Percentage of lymphocytes & 32.2 & $20.0-40.0$ & $\%$ \\
Percentage of neutrophils & 3.8 & $3.0-15.0$ & $\%$ \\
Percentage of neutrophilic granulocytes & 64.0 & $50.0-70.0$ & $\%$ \\
Red blood cell count & 5.04 & $4.30-5.80$ & $10^{12} / 1$ \\
Hemoglobin level & 159 & $130-175$ & $\mathrm{~g} / 1$ \\
Hematocrit level & 45.6 & $40.0-54.0$ & $\%$ \\
Mean corpuscular volume & 90.5 & $80.0-100.0$ & $\mathrm{fl}$ \\
Mean corpuscular hemoglobin & 31.5 & $27.0-34.0$ & $\mathrm{pg}$ \\
Concentration of mean corpuscular hemoglobin & 348 & $320-360$ & $\mathrm{~g} / 1$ \\
Red blood cell distribution width, coefficient & 12.9 & $11.0-16.0$ & $\%$ \\
of variation & & & $\%$ \\
Red blood cell distribution width, standard & 42.5 & $35.0-56.0$ & $10^{9} / 1$ \\
deviation & & & $\mathrm{fl}$ \\
Platelet count & 174 & $125-350$ & $\mathrm{fl}$ \\
Mean platelet volume & 8.2 & $6.5-12.0$ & $\mathrm{fl}$ \\
Platelet distribution width & 15.7 & $9.0-17.0$ & \\
Plateletcrit level & 0.142 & $0.108-0.282$ & \\
\hline
\end{tabular}

Table II. Details of systemic anti-inflammatory treatment administered intravenously for 3 days.

\begin{tabular}{lcc}
\hline Treatment & Dose & Frequency \\
\hline $0.9 \%$ sodium chloride injection & $100 \mathrm{ml}$ Days & Twice daily \\
Clindamycin phosphate for injection & $0.6 \mathrm{~g}$ & Twice daily \\
$5 \%$ glucose and sodium chloride injection & $250 \mathrm{ml}$ & Once daily \\
Vitamin C injection & $2 \mathrm{~g}$ & Once daily \\
Dexamethasone phosphate sodium injection & $5 \mathrm{mg}$ & Once daily \\
Ornidazole and sodium chloride injection & $0.5 \mathrm{~g}$ & Once daily \\
\hline
\end{tabular}

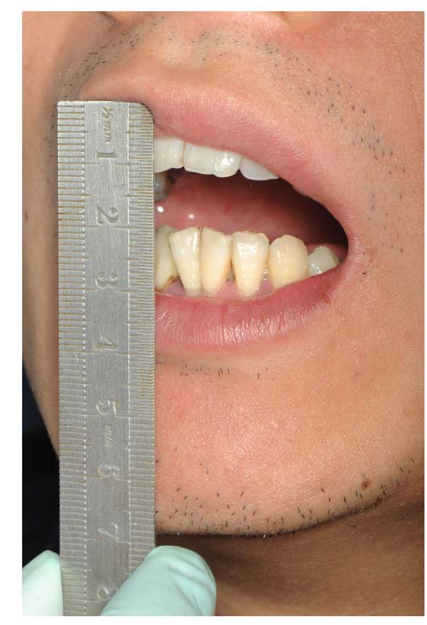

Figure 3. The extent of mouth opening was approximately $10 \mathrm{~mm}$ after 3 days of systemic anti-inflammatory treatment (33 days post-operatively). was cleaned with normal saline. The patient was instructed to perform mouth-opening training with a hot compress and physiotherapy. Finally, the patient was provided oral hygiene education and asked to return for clinical follow-up when he experienced discomfort mainly due to slight trismus.

At 30 days post-operatively, the patient presented with trismus which had not disappeared; it had gradually worsened after self-opening training. A clinical examination revealed that the wound in the area of tooth 38 was covered with food residue; the surrounding soft tissue was red and swollen. There was no sign of pyorrhea in the area of tooth 38. The buccal mucosa was swollen and a sensation of motion was not obvious. The extent of mouth opening was approximately $2 \mathrm{~mm}$ (Fig. 2A), indicating more severe trismus; this was accompanied by slight pain under the left jaw on palpation of swollen lymph nodes. No obvious abnormalities were observed in a routine blood examination 

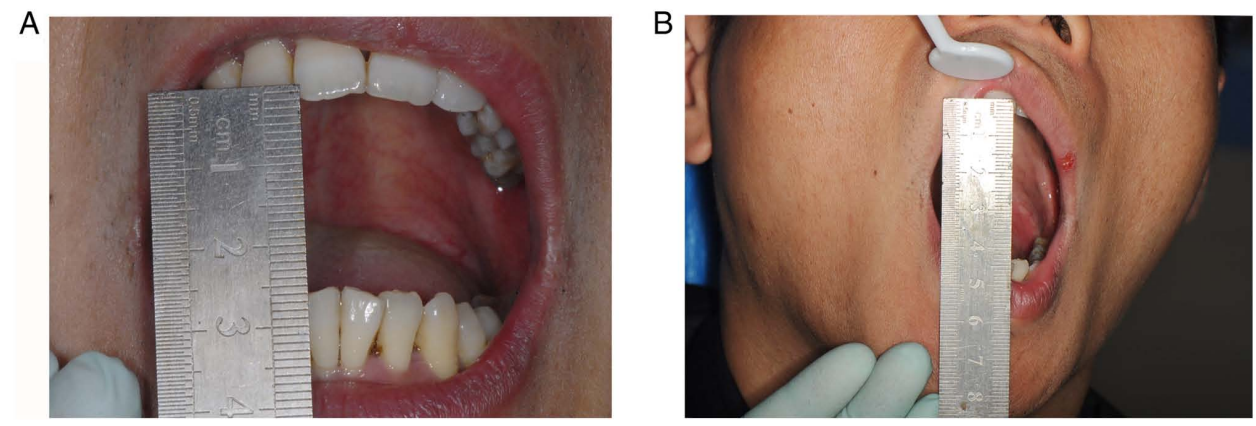

Figure 4. (A) The extent of mouth opening was approximately $25 \mathrm{~mm}$, indicating slight limited mouth opening. (B) An occlusal pad was placed to achieve compulsory mouth opening and the extent of passive mouth opening was approximately $38 \mathrm{~mm}$.
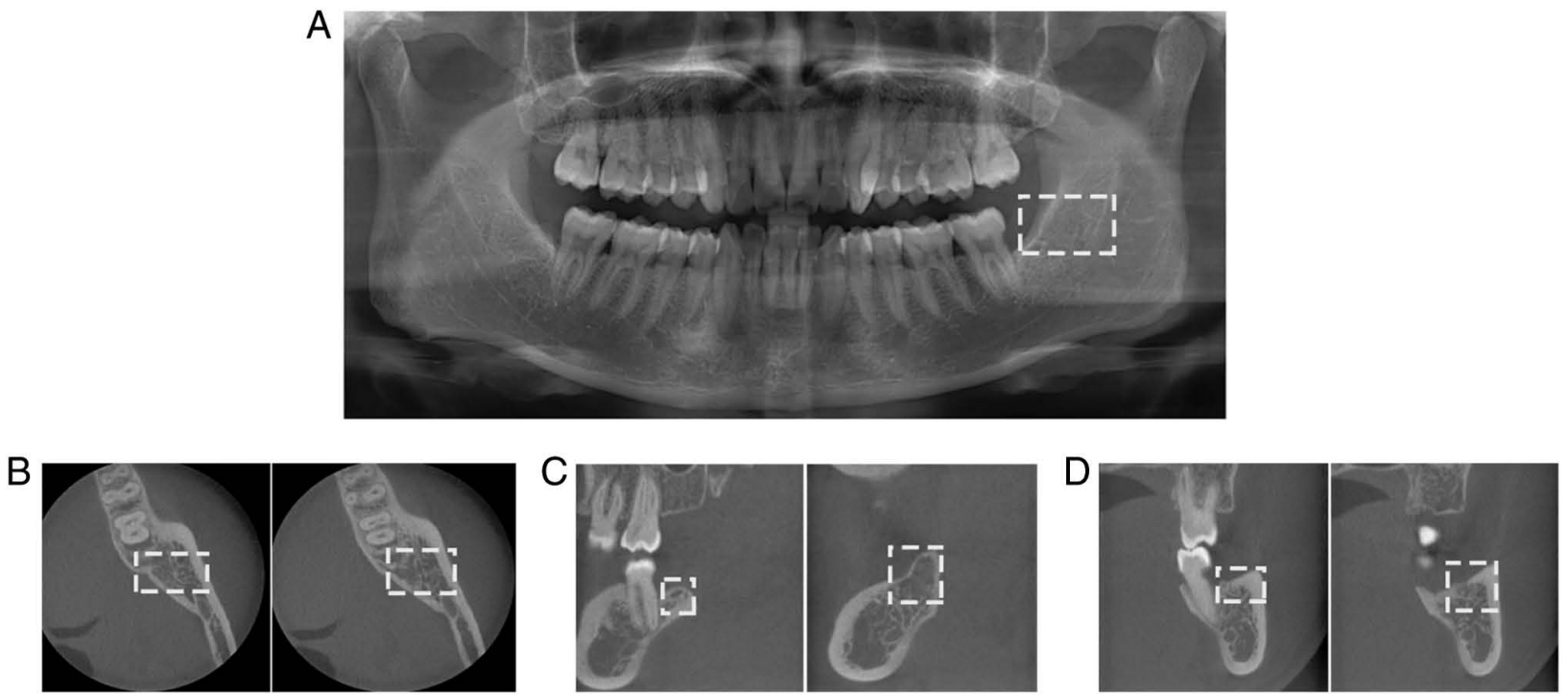

Figure 5. (A) Panoramic X-ray imaging (white rectangular region) and (B-D) cone beam computed tomography (white rectangular region) revealed new bone formation in the alveolar fossa within the area of tooth 38 at 1 year post-operatively.

(Table I). Panoramic X-ray imaging demonstrated reduced bone density in the left mandible (Fig. 2B, white rectangular region) and tight occlusion of anterior teeth without a gap. Cone beam computed tomography (CBCT) could not clearly discern the repair to bone in the alveolar fossa of tooth 38 (Fig. 2C-E). The lingual bone plate in the root tip area was missing (Fig. $2 \mathrm{C}$ and $\mathrm{D}$, white arrows) and the 3-dimensional reconstruction of the CBCT images also revealed the absence of the lingual bone plate (Fig. 2F and G, white rectangular region); moreover, obvious lamellar periosteal hyperplasia was present in the corresponding area (Fig. 2D and E, red arrows). Some areas of the lingual bone plate were slightly rough (Fig. 2E, white rectangular region), and the soft tissue gap was difficult to discern in the bottom of the left side of the mouth. These radiological features indicated the marginal osteomyelitis of the left mandible. As the sensation of motion was not obvious and the patient exhibited severe trismus, no surgery could be performed inside the mouth. Therefore, the following local treatment was applied: The area of tooth 38 was cleaned with hydrogen peroxide and normal saline; subsequently, systemic anti-inflammatory treatment was administered intravenously for 3 days (Table II).
Following 3 days of systemic anti-inflammatory treatment, there was a slight improvement in the trismus symptom of the patient; edema and pain had faded in the left submandibular region, and the extent of mouth opening was approximately $10 \mathrm{~mm}$ (Fig. 3). There was an obvious motion sensation in the area of tooth 38, although no evacuation from the periodontium or other fistulas was observed. Under local infiltration anesthesia with methylpiperazine hydrochloride/epinephrine, the oral mucosa in the area of tooth 38 was treated with incision and drainage, causing discharge of a large volume of bloody exudate. Inflammatory tissue was scraped from the wound; considerable volumes of ornidazole and sodium chloride injection, as well as normal saline, were used to clean and wash the wound in an alternating manner.

Resolution of trismus. At 42 days post-operatively, the patient returned for follow-up and presented alleviation of trismus. The extent of mouth opening was approximately $25 \mathrm{~mm}$, indicating slight trismus (Fig. 4A). The soft tissue demonstrated healing without any sign of pain after palpation. An occlusal pad was placed to achieve compulsory mouth opening; the extent of passive mouth opening was approximately $38 \mathrm{~mm}$ (Fig. 4B). At 1 year post-operatively, the patient presented no discomfort. 
No obvious abnormalities were found in clinical examination. Panoramic X-ray imaging (Fig. 5A, white rectangular region) and CBCT (Fig. 5B-D, white rectangular regions) revealed new bone formation in the alveolar fossa within the area of tooth 38 .

\section{Discussion}

The extraction of third molars can easily lead to tissue injury, inflammation and other post-operative complications, among which trismus is one of the most common (1). It is closely related to post-operative edema, and is typically substantially alleviated or disappeared within 1 to 2 weeks post-operatively. A number of factors may contribute to the onset of trismus. For example, during the extraction of mandibular third molar from the buccal side, the muscle tendon may be severed; this painful stimulus causes muscle protection, which results in trismus (2). Moreover, during inferior alveolar nerve block anesthesia, masticatory muscle spasms may be caused by incomplete disinfection and infection. Brooke (6) reported that multiple needle injections during anesthesia may cause inflammation in that area, while the stretching of intraoral muscles and muscle spasms may be elicited by the use of narcotic drugs. However, the symptoms of progressive trismus in the patient presented herein were not greatly affected by the above factors.

Early during the course of the disease, the patient of the present study exhibited mild trismus. However, this did not receive extensive monitoring as it was presumed to be related to postoperative edema and anxiety. However, when more severe trismus was observed in combination with marginal osteomyelitis, the attending physician intervened with a treatment plan for management of marginal osteomyelitis. Due to the delayed diagnosis by the attending physician, the disease treatment was delayed, which affected the patient's post-operative recovery and daily activities. Based on the intraoperative findings, the absence of the lingual bone plate in the apical region was presumed to be the main cause of marginal osteomyelitis. It was hypothesized that the following was the possible cause of trismus: An infection after tooth extraction spreads along the lingual space of the mandible. Inflammatory mediators can cause irritation associated with muscle tendon attachment to the mandibular ramus and the anterior section of the medial pterygoid muscle, thereby resulting in masticatory muscle spasms that lead to trismus.

For the patient in the present study, at 33 days post-operatively, the surgeon performed incision and drainage in the area of tooth 38 to eliminate a large amount of bloody exudate. The progressive trismus was presumed to result from long-term contact between low-toxicity inflammatory exudate and the muscle tendon. Tight suturing had been performed at the end of surgery. Notably, the inflammatory exudate could not drain with sufficient speed; thus, it spread along the lingual space of the mandible and interacted with the medial pterygoid muscle, which caused fascial space infection associated with marginal mandibular osteomyelitis. Treatment of chronic osteomyelitis has been a major focus in the field of maxillofacial surgery; the main sources of chronic osteomyelitis include odontogenic infection and tooth extraction-related infection (7). Bamberger encouraged the use of systemic antibiotic treatment for
4 weeks post-operatively to manage chronic osteomyelitis; using this approach, surgical treatment was suggested to be unnecessary (8). By contrast, Baur et al (7) reported that surgical treatment was generally more effective than single drug treatment; moreover, conservative drug treatment often leads to multiple recurrences of chronic osteomyelitis. For the patient presented herein, the incision and drainage in the area of tooth 38 achieved ideal results by combination with drug treatment; this was potentially due to the fact that the patient was a young adult with robust overall health and the drainage of inflammatory exudate was performed relatively rapidly. Importantly, long-term follow-up was necessary for our patient.

Tolstunov et al (9) found that the thickness of the lingual bone plate of mesial or horizontal impacted mandibular third molars was thinner than that of vertical impacted mandibular third molars; thus, they stated that the lingual bone plate should be protected during tooth extraction procedures. Lang et al (10) reported that the post-operative use of antibiotics could effectively reduce the incidence of infection. If tooth extraction causes extensive trauma and a large volume of blood is lost, a drainage strip should be placed in the wound for 1 day to prevent infection. This approach also may aid reducing postoperative edema of the patient's cheek. However, some studies have demonstrated that when the mandibular third molar is tilted to the lingual side, less post-operative inflammation will occur when the lingual approach is used to remove the lingual bone plate, compared with the buccal approach; thus, edema and pain will be significantly reduced $(11,12)$. Accordingly, during the extraction of impacted third mandibular molars, protection of the soft and hard tissues around the buccal and lingual sides should be prioritized and trauma should be minimized to avoid unnecessary injury. When fracture or loss of a lingual bone plate occurs, close monitoring should be performed during follow-up to ensure timely management of symptoms.

In conclusion, the extraction of an impacted mandibular third molar is a very common oral surgery procedure. During the surgery, the surgical area should be protected, and careful assessment is necessary regarding fracture or loss of the lingual bone plate. Effective drainage of inflammatory exudates should be performed to prevent muscle spasms and trismus caused by chronic contact with low-toxicity inflammatory exudates.

\section{Acknowledgements}

Not applicable.

\section{Funding}

The present study was supported by the Sun Yat-Sen Scientific Research Launch Project (grant no. YXQH201901), the Natural Science Foundation of Guangdong Province, China (grant no. 2018A0303130106 and 2018A030313759).

\section{Availability of data and materials}

The data used during the present study are available from the corresponding author on reasonable request. 


\section{Authors' contributions}

YZ, PZ, BJ, ZW and ZZ conceived the case report, wrote the initial manuscript and reviewed the final manuscript. JX, QC, LN, ZW and ZZ interpreted and created the clinical and radiographic images, and reviewed the final manuscript. All authors read and approved the final manuscript.

\section{Ethics approval and consent to participate}

The patient provided informed consent for his involvement in the present study.

\section{Patient consent for publication}

The patient provided informed consent for the publishing of the relevant clinical data.

\section{Competing interests}

The authors declare that they have no competing interests.

\section{References}

1. Bui $\mathrm{CH}$, Seldin EB and Dodson TB: Types, frequencies, and risk factors for complications after third molar extraction. J Oral Maxillofac Surg 61: 1379-1389, 2003.

2. Balakrishnan G, Narendar R, Kavin T, Venkataraman S and Gokulanathan S: Incidence of trismus in transalveolar extraction of lower third molar. J Pharm Bioallied Sci 9 (Suppl 1): S222-S227, 2017.
3. Gülnahar Y and Kupeli I: Effect of preemptive intravenous ibuprofen on postoperative edema and trismus in third molar tooth extraction: A randomized controlled study. J Dent Anesth Pain Med 18: 161-167, 2018.

4. Li C, Men Y and Li L: Discrepancies on dexamethasone for trismus after third molar extraction. Oral Surg Oral Med Oral Pathol Oral Radiol 117: 253, 2014.

5. Steed MB: The indications for third-molar extractions. J Am Dent Assoc 145: 570-573, 2014.

6. Brooke RI: Postinjection trismus due to formation of fibrous band. Oral Surg Oral Med Oral Pathol 47: 424-426, 1979.

7. Baur DA, Altay MA, Flores-Hidalgo A, Ort Y and Quereshy FA: Chronic osteomyelitis of the mandible: Diagnosis and management-an institution's experience over 7 years. J Oral Maxillofac Surg 73: 655-665, 2015.

8. Bamberger DM: Diagnosis and treatment of osteomyelitis. Compr Ther 26: 89-95, 2000.

9. Tolstunov L, Brickeen M, Kamanin V, Susarla SM and Selvi F: Is the angulation of mandibular third molars associated with the thickness of lingual bone? Br J Oral Maxillofac Surg 54: 914-919, 2016.

10. Lang MS, Morrow AJ, Gonzalez ML and Dodson TB: Do postoperative antibiotics decrease the frequency of inflammatory complications following third molar removal? J Oral Maxillofac Surg 74: e33-e34, 2016.

11. Singh V, Alex K, Pradhan R, Mohammad S and Singh N: Techniques in the removal of impacted mandibular third molar: A comparative study. Eur J Gen Dent 2: 25-30, 2013.

12. Yang TJ, Li Y and Zhu HY: Removal of lingual bone plate in the extraction of lower wisdom teeth. J Oral Maxillofac Surg 24: 63, 2014.

This work is licensed under a Creative Commons Attribution-NonCommercial-NoDerivatives 4.0 International (CC BY-NC-ND 4.0) License. 